\section{A Systematic Survey of Control Groups in Behavioral and Social Science Trials}

Research on Social Work Practice

2018, Vol. 28(5) 538-545

(C) The Author(s) 2017

Reprints and permission:

sagepub.com/journalsPermissions.nav DOI: I0.II77/I04973/5I77/8940

journals.sagepub.com/home/rsw

(S)AGE

\author{
Mei Wang ',2, Guangwen Sun ${ }^{3}$, Yaping Chang', Yanling Jin', \\ Alvin Leenus ${ }^{4}$, Muhammad Maaz ${ }^{4}$ Guowei $\mathrm{Li}^{1,2}$, Meha Bhatt', \\ Luciana P. F. Abbade ${ }^{5}$, Ikunna Nwosu', Laura Zielinski ${ }^{6}$, \\ Nitika Sanger ${ }^{7}$, Bianca Bantoto ${ }^{8}$, Candice Luo ${ }^{9}$, leta Shams ${ }^{2,10,11}$ \\ Hamnah Shahid $^{12}$, Jonathan Adachi ${ }^{13}$, Lawrence Mbuagbaw ${ }^{1,2,14}$, \\ Mitchell Levine $^{1,13}$, Zainab Samaan ${ }^{1,15}$, and Lehana Thabane ${ }^{1,11}$
}

\begin{abstract}
Behavioral and social sciences randomized controlled trials (BSSTs) have a significant role in life sciences. Choosing an appropriate control or comparator group for BSSTs is critical, to provide true intervention effects. The objective of this study was to determine the types of control groups used in BSSTs, and the rationale provided to justify these choices. We conducted a systematic survey of BSST protocols published between January 2012 and October 2016 in the Cochrane Library and Medline databases. We randomly selected 200 protocols. The study selection and data extraction were performed independently in duplicate. The most frequent control groups were active concurrent $(97 / 200,48.5 \%)$, and no treatment concurrent controls (88/200, 44.0\%). The majority of studies (7I.5\%) did not provide justification for comparators choice. We concluded that BSSTs trials compare interventions to active and no treatment controls however the majority of trials lacked rationale for the selection of the study comparator.
\end{abstract}

\title{
Keywords
}

systematic survey, comparator, behavioral and social sciences research

Behavioral and social sciences randomized control trials (BSSTs) aim to evaluate the effects of specific interventions (social or behavioral processes, or the use of these processes) on health-related biomedical or behavioral outcomes (McGrath, 1994). Behavioral and social factors are significant predictors of health problems. The interaction of biological, behavioral, and social factors are closely related to health and illness (Anderson, 1997). Therefore, BSSTs have an important role in modern life sciences. However, the methods employed in these types of clinical trials can be complicated since behavioral and social interventions as well as their outcomes will often include various components. For instance, these interventions might involve enhancing motivation, which may incorporate the use of activity tracking devices; encouraging physical activity, which may involve incentives and rewards; and improving participants' quality of life, which may incorporate coping skills, acceptance, and engagement with important aspects of life (Marsden et al., 2017; Samaan et al., 2015).

The control or comparator group in a clinical trial is an essential element of the research question and architecture (Dawson et al., 2009; Sackett \& Wennberg, 1997). The ethical integrity and validity of the trial are contingent upon the correct choice of control or comparator group (Brigham, Feaster,
'Department of Health Research Methods, Evidence, and Impact (HEI), McMaster University, Hamilton, Ontario, Canada

${ }^{2}$ Father Sean O'Sullivan Research Institute, McMaster University, Hamilton, Ontario, Canada

${ }^{3}$ Dundas Valley Secondary School, Dundas, Ontario, Canada

${ }^{4}$ Faculty of Health Sciences, McMaster University, Hamilton, Ontario, Canada

${ }^{5}$ Department of Dermatology and Radiotherapy, Botucatu Medical School, Universidade Estadual Paulista, UNESP, São Paulo, Brazil

${ }^{6}$ McMaster Integrative Neuroscience Discovery and Study, McMaster University Hamilton, Ontario, Canada

${ }^{7}$ Medical Sciences, McMaster University Hamilton, Ontario, Canada

${ }^{8}$ Integrated Sciences, McMaster University, Hamilton, Ontario, Canada

${ }^{9}$ Health Sciences, McMaster University, Hamilton, Ontario, Canada

${ }^{10}$ Psychology, Neuroscience and Behaviour, McMaster University, Hamilton, Ontario, Canada

"Centre for Evaluation of Medicine, St. Joseph's Healthcare Hamilton, Hamilton, Ontario, Canada

${ }^{12}$ Arts and Science, McMaster University, Hamilton, Ontario, Canada

${ }^{13}$ Department of Medicine, McMaster University, Hamilton, Ontario, Canada

${ }^{14}$ Centre for Development of Best Practices in Health, Yaoundé Central Hospital, Yaoundé, Cameroon

${ }^{15}$ Department of Psychiatry and Behavioural Neurosciences, McMaster University, Hamilton, Ontario, Canada

\section{Corresponding Author:}

Lehana Thabane, Biostatistics Unit, Father Sean O'Sullivan Research Centre, 3rd Floor Martha, Room H325, St. Joseph's Healthcare Hamilton, 50 Charlton Avenue East, Hamilton, Ontario, Canada L8N 4A6.

Email: thabanl@mcmaster.ca 
Wakim, \& Dempsey, 2009; World Medical Association, 1997). Recently, the National Institutes of Health (NIH) released an updated policy aimed at improving the quality and efficiency of clinical trials (Hudson, Lauer, \& Collins, 2016). It included some guidelines for choosing appropriate comparators in clinical trials (Center for Medical Technology Policy, 2010; European Medicines Agency, 2001; Food and Drug Administration, 2001). While applicable to some extent, these guidelines do not adequately address all BSSTs. The choice of an inappropriate comparator may lead to either over- or underestimation of the interventions' true effects (Kelly, Davies, Saint-Raymond, Tomasi, \& Offringa, 2016). Given the limited guidance on how to choose (a) control intervention(s) in BSSTs, we attempted to fill this knowledge gap by collecting information pertaining to the current practices investigators use to select their control intervention(s).

The objective of this systematic survey was to determine the types of control groups or comparators used in BSSTs and to investigate the rationale used to justify these choices.

\section{Method}

\section{Search Strategy and Eligibility Criteria}

In order to address the research question about the justification of comparator selection, we opted to include study protocols instead of final trial reports since protocols provide more details on the methodology and rationale. To achieve this goal, we conducted a systematic survey of BSST protocols published between January 2012 and October 2016 in the Cochrane Library and Medline databases (see Online Appendix A). We searched for additional references by cross-checking bibliographies of relevant reviews (Brigham et al., 2009; Campbell et al., 2012). This time frame was chosen to place emphasis on recently published protocols since they tend to have greater transparency and reproducibility of study results when compared to protocols published in earlier years (Chan et al., 2014).

The search strategy included terms for randomized controlled trials (RCTs) [random $\$$ adj3 trial\$] [random $\$$ adj3 stud\$], protocol, behavior [behav\$], social sciences [soci\$ adj3 science\$], and a time limit (January 2012 to December 2016; see Online Appendix A). After identifying the BSST protocols, we assigned numbers sequentially to each one and used an online random sequence generator to randomly select 200 protocols (https://www.random.org/integers/). We randomly selected a subset of the protocols in an attempt to maintain the feasibility of the study.

We included protocols of RCTs in which the intervention(s) included behavioral or social component(s) or the main outcome(s) targeted behavioral or social outcomes (see Online Appendix B for the possible components of behavior and social sciences interventions and outcomes; Michie, van Stralen, \& West, 2011). Only studies which involved human subjects and were written in English were included. Exclusion criteria included systematic reviews, nonrandomized studies, and conference abstracts.
Two independent investigators (MW and GS) performed the selection process in two phases - title and abstract screening, followed by random selection of 200 protocols for full-text review (see Figure 1). Any articles that did not meet the inclusion criteria during the screening or review phase were replaced by other protocols from the random selection list. Disagreements were resolved by consensus or by consulting a third author.

\section{Data Extraction}

Six reviewers (MW, YC, GS, YJ, AL, MM), with training in methodology, independently extracted the data using a data collection Excel form, which had been pilot tested on 20 identified protocols beforehand.

To obtain data on study characteristics, the following information was collected: (1) surname of first author, (2) name of journal, (3) publication year, (4) study design, (5) type of participants, (6) experimental intervention(s), (7) control intervention(s), (8) primary outcome(s), (9) sample size, (10) country, (11) setting (health care vs. nonhealth care) (12) number of sites (multi vs. single) (13) number of arms, (14) funding source, (15) randomization level (at individual level vs. at cluster level), (16) randomization techniques, (17) method of randomization, (18) method of the allocation concealment, (19) blinding or not, and (20) blinded parties (participants, investigators, data extractors, care providers).

To assess the current evidence for selecting comparator groups, answers to the following questions were collected: (1) what is the primary objective? (2) Which kind of study design did the author choose? (3) Which kinds of control conditions did the author choose? (4) What is the choice of comparator group? (5) If placebo (or no treatment) was used as a comparator, what was the rationale for the choice? (6) If a standard of care or usual care was used as a comparator, what was the rationale for the choice? (7) If an active therapy was used as a comparator, what was the rationale for the choice? (8) Comment on comparator justification or consideration for alternative selection (see Online Appendix C).

\section{Data Analysis}

A discussion and analysis of the current practices used by authors of the included protocols to select comparator groups were carried out based on the data extracted. Statistics for individual items were calculated and presented as percentages. We created a list of justification criteria to address the choice of comparator group(s) based on the systematic survey findings and a literature review of existing guidelines on the conduct of BSSTs.

\section{Results}

\section{Search and Studies}

A total of 2,598 articles were found from searching electronic databases and additional sources, and after excluding 


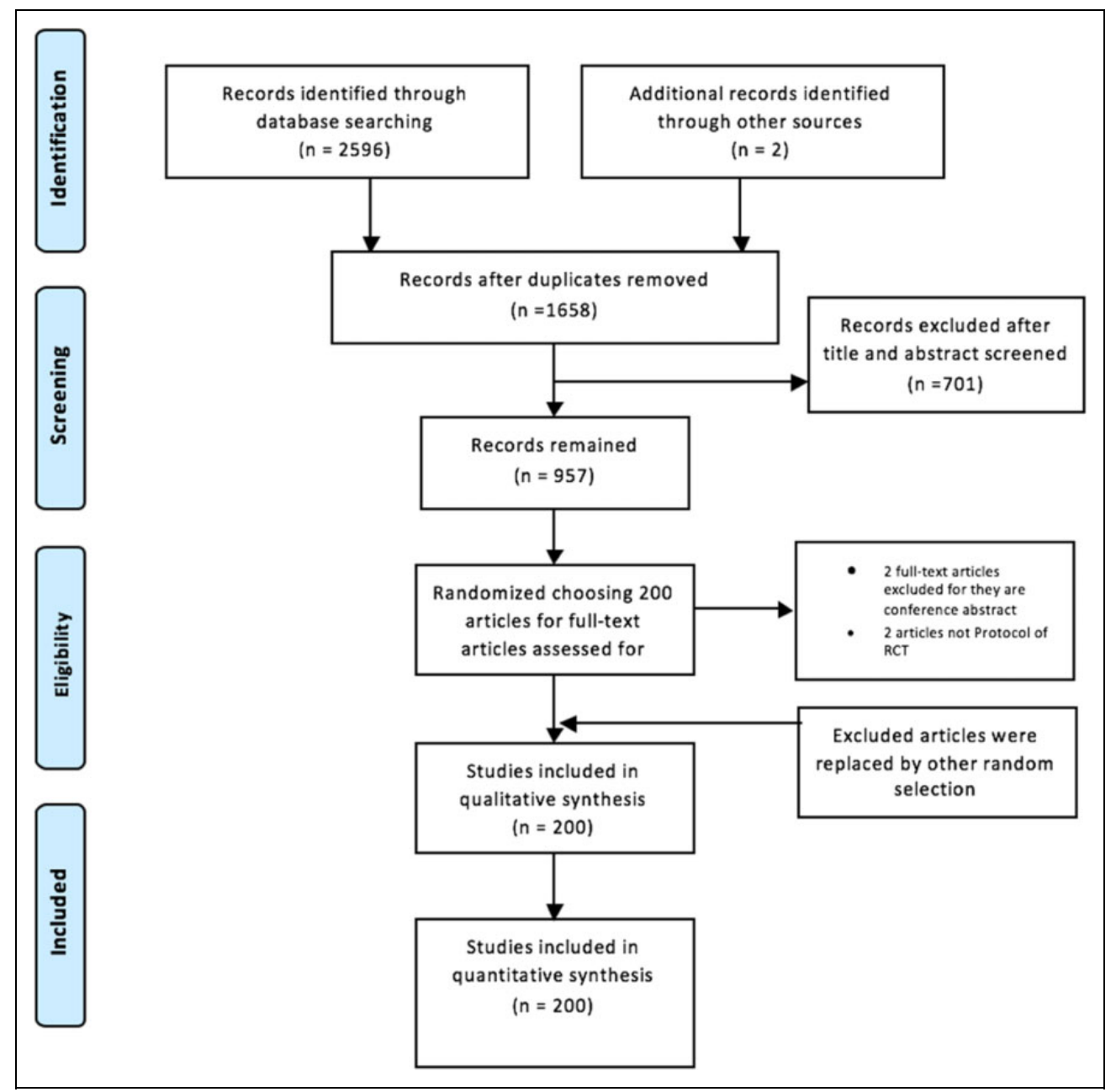

Figure I. Preferred Reporting Items for Systematic Reviews and Meta-Analyses (PRISMA) flow diagram for the study selection process.

duplicates and screening titles and abstracts, we were left with 957 protocols. We excluded 4 of the first 200 randomly selected protocols and replaced them with 4 others that were randomly chosen from the remaining 957 protocols (see Figure 1). The list of the 200 protocols used for quantitative synthesis can be found in Online Appendix D.

\section{Characteristics of the Studies}

The 200 included protocols were published in 29 distinct journals, with nearly half $(43.5 \%)$ having been published in the journal Trials (see Table 1). The majority of the studies were planned to be conducted in Europe (51.5\%), followed by America (20.5\%), Australia-Oceania (17.0\%), Asia-Middle East (7.0\%), and lastly Africa (1.5\%) or multicontinent $(2.5 \%)$. More than half $(58.5 \%)$ of the BSSTs were planned to be conducted in a health-care setting. Seventy-nine $(39.5 \%)$ studies planned to include multiple study sites. Most of the studies were two-arm studies $(80.0 \%)$, and $91.5 \%$ of the 200 studies were funded by noncommercial sources. Most of those studies $(77.5 \%)$ were superiority studies, and 22 of 200 $(11.0 \%)$ were pilot or feasibility studies. Fifty-three $(26.5 \%)$ studies intended to select their participants from the community, and the rest of the studies (68\%) were focused on a specific population (e.g., breast cancer survivors).

There were $45(22.5 \%)$ studies that planned to randomize participants on cluster level; the other 155 studies planned individual randomization. There were 39 (19.5\%) studies that planned to use block and stratified randomization simultaneously; $50(25.0 \%)$ studies planned to use only stratified randomization; 37 (18.5\%) studies planned to use only blocked randomization; 23 (11.5\%) studies planned to use simple randomization. The remaining $48(24 \%)$ studies did not provide details regarding the randomization technique. About half of the studies $(50.5 \%)$ planned to use computer-generated randomization, and $33(16.5 \%)$ studies planned to use web-based methods to generate a randomization sequence. Fifty two $(26.0 \%)$ studies did not provide details of the randomization method.

In terms of concealment, we found that $62(31.0 \%)$ studies either did not provide information regarding concealment or did not plan for any concealment. With regard to blinding, there were $26(13.0 \%)$ studies that were not blinded and $58(29.0 \%)$ studies that did not provide information about blinding. Of the 
Table I. Characteristics of the 200 Included Protocols.

\begin{tabular}{|c|c|c|}
\hline Characteristic & Category & Number (\%) \\
\hline \multirow[t]{5}{*}{ Journals } & Trials & $87(43.5)$ \\
\hline & BMC Public Health & $33(16.5)$ \\
\hline & BMC Psychiatry & $16(8.0)$ \\
\hline & BMJ Open & $12(6.0)$ \\
\hline & Other 25 journals & $52(26.0)$ \\
\hline \multirow[t]{6}{*}{ Continents } & Europe & $103(5 \mid .5)$ \\
\hline & Americas & $4 I(20.5)$ \\
\hline & Australia-Oceania & $34(17.0)$ \\
\hline & Asia-Middle East & $14(7.0)$ \\
\hline & Multicontinents & $5(2.5)$ \\
\hline & Africa & $3(1.5)$ \\
\hline \multirow[t]{3}{*}{ Type of setting } & Health care & $117(58.5)$ \\
\hline & Nonhealth care & $80(40.0)$ \\
\hline & Mixed & $3(1.5)$ \\
\hline \multirow[t]{2}{*}{ Number of site } & Single site & $121(60.5)$ \\
\hline & Multiple sites & $79(39.5)$ \\
\hline \multirow{3}{*}{ Participants } & Community-wide & $53(26.5)$ \\
\hline & Specific population & $136(68)$ \\
\hline & Clinical subjects & II (5.5) \\
\hline \multirow[t]{2}{*}{ Number of arm } & Two arms & $160(80.0)$ \\
\hline & Multiple arms & $60(20.0)$ \\
\hline \multirow[t]{4}{*}{ Funding source } & Noncommercial & $183(9 \mid .5)$ \\
\hline & No funding & $10(5.0)$ \\
\hline & Mixed funding & $6(3.0)$ \\
\hline & Privacy & $\mathrm{I}(0.5)$ \\
\hline \multirow[t]{6}{*}{ Trial objective } & Superiority & I 55 (77.5) \\
\hline & Pilot or feasibility study & $22(11.0)$ \\
\hline & Noninferiority or equivalence & $18(9.0)$ \\
\hline & Superiority + cost-effectiveness & $2(1.0)$ \\
\hline & Cost-effective & $2(1.0)$ \\
\hline & Noninferiority + cost effective & $\mathrm{I}(0.5)$ \\
\hline \multirow{2}{*}{$\begin{array}{l}\text { Level of } \\
\quad \text { randomization }\end{array}$} & Individual level & I55 (77.5) \\
\hline & Cluster level & $45(22.5)$ \\
\hline \multirow{5}{*}{$\begin{array}{l}\text { Randomization } \\
\text { technique }\end{array}$} & No details given & $48(24.0)$ \\
\hline & Block randomization & $37(18.5)$ \\
\hline & Stratified randomization & $50(25.0)$ \\
\hline & Block and stratified randomization & $39(19.5)$ \\
\hline & Simple randomization & $23(11.5)$ \\
\hline \multirow{6}{*}{$\begin{array}{l}\text { Method of } \\
\text { randomization }\end{array}$} & Computer generated & $101(50.5)$ \\
\hline & No details given & $52(26.0)$ \\
\hline & Web-based system & $33(16.5)$ \\
\hline & Random number tables & $9(4.5)$ \\
\hline & Flipping a coin & $\mathrm{I}(0.5)$ \\
\hline & $\begin{array}{l}\text { Random number tables }+ \\
\text { computer generated }\end{array}$ & $4(2.0)$ \\
\hline \multirow[t]{5}{*}{$\begin{array}{l}\text { Allocation } \\
\text { concealment }\end{array}$} & $\begin{array}{l}\text { No information was given (or no } \\
\text { concealment) }\end{array}$ & $62(31.0)$ \\
\hline & By an independent agent & $61(30.5)$ \\
\hline & Kept centrally & $33(16.5)$ \\
\hline & Opaque/sealed envelope & $27(13.5)$ \\
\hline & Multiple methods & $16(8.0)$ \\
\hline \multirow{5}{*}{ Blinding condition } & Single blind & $87(43.5)$ \\
\hline & No information was given & $58(29.0)$ \\
\hline & Nonblinded & $26(13.0)$ \\
\hline & Double blind & $22(11.0)$ \\
\hline & Multiple blind & $7(3.5)$ \\
\hline
\end{tabular}

(continued)
Table I. (continued)

\begin{tabular}{|c|c|c|}
\hline Characteristic & Category & Number (\%) \\
\hline \multirow[t]{19}{*}{ Blinding party } & Assessor/observer/evaluator & $58(29.0)$ \\
\hline & No information was given & $53(26.5)$ \\
\hline & Nonblinded & $25(12.5)$ \\
\hline & Double blinded & $33(16.5)$ \\
\hline & Assessor + statistician & 12 \\
\hline & Assessor + investigator & 5 \\
\hline & Assessor + research assistant & 5 \\
\hline & Assessor + participants & 3 \\
\hline & Participants + research assistant & 3 \\
\hline & Investigator + statistician & 2 \\
\hline & Assessor + therapist & I \\
\hline & Therapist + research assistant & I \\
\hline & Therapist + investigator & I \\
\hline & Multiblinding $(>2)$ & $9(4.5)$ \\
\hline & Statistician & $9(4.5)$ \\
\hline & Participants & $4(2.0)$ \\
\hline & Research assistant & $4(2.0)$ \\
\hline & Investigator & $3(1.5)$ \\
\hline & Therapist/clinician & $2(1.0)$ \\
\hline \multirow[t]{4}{*}{ Analysis Basis } & Intention to treat & $87(43.5)$ \\
\hline & No information was given & $88(44.0)$ \\
\hline & Intention to treat + per protocol & $22(11.0)$ \\
\hline & Per protocol & $3(1.5)$ \\
\hline
\end{tabular}

Table 2. Type of Comparator Choice in 200 Protocols.

\begin{tabular}{lc}
\hline Comparator Choice & Number of Trials (\%) \\
\hline No treatment concurrent control & $88(44.0 \%)$ \\
Placebo concurrent control & $4(2.0 \%)$ \\
Different regimen concurrent control & $4(2.0 \%)$ \\
Active treatment concurrent control & $97(48.5 \%)$ \\
Multiple control groups & $7(3.5 \%)$ \\
Historical control & 0 \\
Total & 200 \\
\hline
\end{tabular}

142 studies that supplied information about blinding, single blinding was the most common (87 studies, $43.5 \%$ ).

\section{The Types and Condition of Control Group Intervention}

Generally, the following types of controls are recognized in RCTs: (1) placebo concurrent control, (2) dose-comparison concurrent control, (3) no treatment concurrent control, (4) active treatment concurrent control, (5) and historical control (European Medicines Agency, 2001; Food and Drug Administration, 2001; see Table 2). We found active concurrent controls in $97(48.5 \%)$ studies and no treatment concurrent control in $88(44.0 \%)$ studies.

There were $193(96.5 \%)$ studies that had a single control group, and only 7 (3.5\%) studies had multiple control groups (see Figure 2). For the studies with a single control group, there 


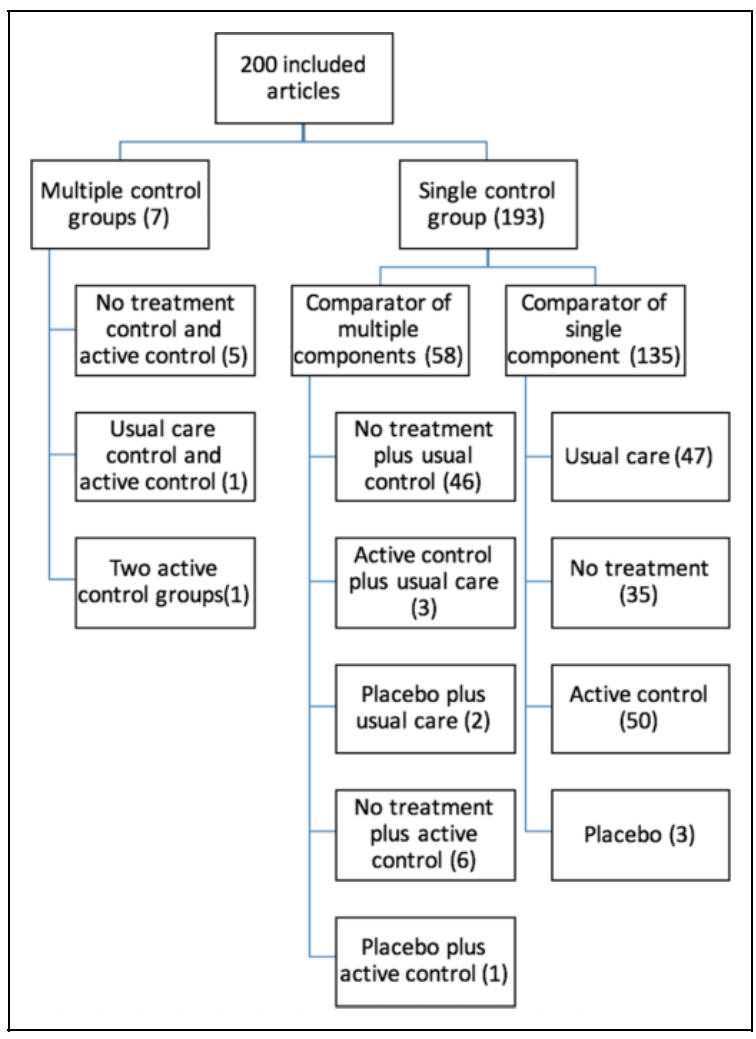

Figure 2. Flow diagram for comparator categories.

were $135(67.5 \%)$ that used a single component comparator, which included no treatment, usual care, placebo, and active control (see Table 3).

The justifications for the selection of the comparator in the 200 included articles can be found in Table 4. For usual care, we found that $73(73 / 99,71.7 \%)$ protocols did not provide justification for the comparators. The most common rationale (48/92, $52.2 \%)$ for choosing no treatment as a component of the comparator was "testing add-on treatment to standard therapy when all subjects in the trial receive all treatments that would typically be prescribed." However, there were still 36 (36/92, $39.1 \%$ ) studies that provided no rationale. For active control, no justification was given in $30(30 / 68,44.1 \%)$ studies, but we found there were $16(16 / 68,23.5 \%)$ studies that justified the choice using "available evidence from at least one RCT published in a peer-reviewed journal." For the six placebo comparator, four of them did not supply any justification; the other two used the "testing add-on treatment" rationale.

In brief, there were a total of 143 studies that did not supply justification for the comparators.

\section{Discussion}

The choice of comparator for an RCT is largely driven by the key research question that the trial is designed to answer. Assurance of clinical equipoise and the proper comparator selection is critical (Mann \& Djulbegovic, 2003). A general characteristic of BSSTs is that most are pragmatic, with the aim of answering the practical question of whether offering an intervention compared with some alternative (e.g., usual care) in routine health care will do more good than harm (Schwartz \& Lellouch, 1967).

\section{Main Findings}

We found that while the 200 randomly selected protocols described studies that were planned to be carried out all over the world, most of them were in Europe (51.5\%) and America $(20.5 \%)$, indicating that BSSTs are most commonly conducted in developed countries. Furthermore, because BSSTs are

Table 3. Condition of Comparator Choice in 200 Protocols.

\begin{tabular}{lcccc}
\hline & & & Acted as control group or one component of a \\
control group
\end{tabular}


Table 4. Justification for Comparator Choice in the 200 Included Articles.

\begin{tabular}{|c|c|c|}
\hline $\begin{array}{l}\text { Comparator or Component } \\
\text { of Comparator }\end{array}$ & Justification Condition & Frequency (\%) \\
\hline Usual care (99) & $\begin{array}{l}\text { I. Commonly used therapy in jurisdiction of trial (evidence based) } \\
\text { 2. Available Meta-analysis / Cochrane systematic review } \\
\text { 3. Available Evidence from at least one RCT published in a peer reviewed journal } \\
\text { 4. Follows Clinical Practice Guidelines (National/other) recommendations } \\
\text { 5. 5. Experience based } \\
\text { 6. No justification given }\end{array}$ & 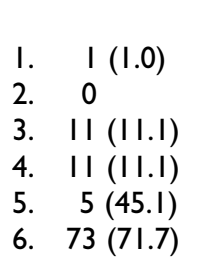 \\
\hline No treatment (92) & $\begin{array}{l}\text { I. There is no standard treatment } \\
\text { 2. Evidence has risen creating substantial doubt regarding the net therapeutic advantage of } \\
\text { standard therapy } \\
\text { 3. Effective treatment is not available to patients due to cost constraints or short supply } \\
\text { 4. In a population of patients who are refractory to standard treatment and for whom no } \\
\text { standard second-line treatment exists } \\
\text { 5. Testing add-on treatment to standard therapy when all subjects in the trial receive all } \\
\text { treatments that would normally be prescribed } \\
\text { 6. Patients have provided an informed refusal of standard therapy for a minor condition for } \\
\text { which patients commonly refuse treatment and when withholding such therapy will not lead } \\
\text { to undue suffering or the possibility of irreversible harm of any magnitude } \\
\text { 7. No rationale given }\end{array}$ & 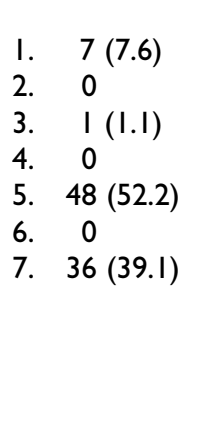 \\
\hline Active control $(68 / 67)$ & $\begin{array}{l}\text { I. Available meta-analysis/systematic review } \\
\text { 2. Available Evidence from at least one RCT published in a peer-reviewed journal } \\
\text { 3. Experience based } \\
\text { 4. Follows Clinical Practice Guidelines (National/other) recommendations } \\
\text { 5. No justification given }\end{array}$ & $\begin{array}{l}\text { I. } \quad 9(13.2) \\
\text { 2. } 16(23.5) \\
\text { 3. } \quad 6(8.8) \\
\text { 4. } \quad 7(10.3) \\
\text { 5. } \quad 30(44.1)\end{array}$ \\
\hline$(6)$ & $\begin{array}{l}\text { I. There is no standard treatment } \\
\text { 2. Standard therapy has been shown to be no better than placebo } \\
\text { 3. Evidence has risen creating substantial doubt regarding the net therapeutic advantage of } \\
\text { standard therapy } \\
\text { 4. Effective treatment is not available to patients due to cost constraints or short supply } \\
\text { 5. In a population of patients who are refractory to standard treatment and for whom no } \\
\text { standard second-line treatment exists } \\
\text { 6. Testing add-on treatment to standard therapy when all subjects in the trial receive all } \\
\text { treatments that would normally be prescribed } \\
\text { 7. Patients have provided an informed refusal of standard therapy for a minor condition for } \\
\text { which patients commonly refuse treatment and when withholding such therapy will not lead } \\
\text { to undue suffering or the possibility of irreversible harm of any magnitude } \\
\text { 8. No rationale given }\end{array}$ & $\begin{array}{ll}\text { I. } & 0 \\
\text { 2. } & 0 \\
\text { 3. } & 0 \\
\text { 4. } & 0 \\
\text { 5. } & 0 \\
6 . & 2(33.3) \\
\text { 7. } & 0 \\
\text { 8. } & 4(66.7)\end{array}$ \\
\hline
\end{tabular}

seldom used in evaluating pharmaceuticals and are always related to public health, social, and behavioral aspects of life, the majority of the studies $(91.5 \%)$ were funded by noncommercial sources.

Randomization is a technique that allows researchers to control for all known and unknown factors that may contribute to the results in treatment groups and comparator(s) (Kim \& Shin, 2014). With the development of computer technology, randomization is becoming easier than before. Accordingly, we found that more than half of the studies $(67.0 \%)$ planned to use a computer-generated method or a web-based method to generate their randomization sequence. However, 52 (26.0\%) protocols did not provide the details of the randomization method. Without effective randomization, even with a perfect choice of comparator, the validity of a study is questionable. Another important component of the randomization process is concealment of the allocation sequence. We found that 62 (31.0\%) studies either did not provide information about their chosen concealment technique or did not plan for concealment. Schulz, Chalmers, Hayes, and Altman (1995) found that studies with inadequate or unclear allocation concealment yielded larger estimates of effect than those with adequate concealment.

For BSSTs, the blinding of participants and/or therapists is never possible, as the behavioral and social science components of the interventions are unlike pharmaceutical interventions, where a dummy placebo can be used. With that being said, the assessor(s) or statistician(s) may still be blinded. In this survey, 58 studies did not provide information about blinding, and 26 studies indicated the impossibility for blinding, which will bias the assessment of outcomes such that no analytical techniques can correct for this limitation (Karanicolas, Farrokhyar, \& Bhandari, 2010). 
In this study, we found that none of the protocols chose historical controls. The most frequent choices for control types were active concurrent control $(48.5 \%)$ and no treatment concurrent control (44.0\%). Furthermore, we found the condition of comparator to be diverse in BSSTs. Although the majority of studies $(96.5 \%)$ chose a single control group as a comparator, there were still 7 (3.5\%) studies that chose multiple control groups as comparators. For single control group, the majority of them $(135 / 193,69.9 \%)$ planned to use traditional single component comparator, and the other studies chose multiplecomponent comparator.

Usual care (one kind of active treatment) is the favorite comparator among researchers designing BSSTs. Acting as a comparator or component of comparator, usual care was found in $99(49.5 \%)$ studies, often appearing under numerous synonymous terms (e.g., standard treatment, care as usual, standard care, treatment as usual, standard of care). In addition, Smelt, van der Weele, Blom, Gussekloo, and Assendelft (2010) reported that the description of instructions and information provided for usual care was often insufficient, which made evaluation of the trials difficult. In this study, we found the rationale for choosing usual care to be suboptimal, as there were $73(73 / 99,71.7 \%)$ studies that provided no justification for selecting this comparator.

Other than usual care, there were 68 different active treatments that were treated as a comparator(s) or component of comparators in 67 studies. There was adequate justification provided in $38(38 / 68,55.9 \%)$ of these studies. However, the other 30 studies did not provide rationale for selecting the active comparator, a shortcoming that will influence the quality of the trials.

The rationale used most often for the no treatment comparator was "testing add-on treatment to standard therapy when all subjects in the trial receive all treatments that would normally be prescribed" (48/92, $52.2 \%)$, the same condition can be found in the placebo choice $(2 / 6,33.3 \%)$. There were $36(36 / 92$, $39.1 \%)$ studies of the no treatment comparator and $4(4 / 6$, $66.7 \%$ ) studies of placebo comparator with no justification, which not only will affect the validity of the studies but also raise ethical concerns.

\section{Strengths and Limitations}

Our study is the first survey to analyze the choice of comparator groups in BSSTs. We performed a systematic search for protocols of BSSTs, which we believe contain more rationale details than trial reports. Furthermore, we provided precise definitions for most of the variables of interest, increasing the reproducibility of our methods. Additionally, all screening results, data extraction, eligibility decisions, and data analysis were carried out in duplicate-having multiple reviewers review the data ensures the accuracy of data extraction and the quality of assessments.

One of the potential limitations of our study design is that we randomly chose only 200 protocols in the past 5 years, which might not represent all the characteristics of comparator choice for BSSTs.

\section{Implications}

The choice of comparator(s) should be carefully selected and justified to provide consistency and reproducibility for BSSTs. This study provides a helpful contribution for the choice of comparator(s) for BSSTs, as no previous review has provided a systematic assessment of comparator(s) types and justification of comparator(s) choice in these trials.

\section{Conclusion}

The most common choices for comparator in BSSTs in the past 5 years were active and no treatment/intervention concurrent controls, and the majority of the studies did not provide the rationale for the selection of the study comparator.

\section{Authors' Note}

MW was responsible for the search strategy, designing, testing of the data extraction form, and writing the initial draft. LM and ZS provided input for the design, analyses and reporting and contributing to the final draft. LT was responsible for the conception, designing of the survey and critical review the final draft. GS, YC, YJ, AL and $\mathrm{MM}$ were involved in designing and testing of the data extraction form. MB, LP, LN, LZ, NS, BB, CL, IS, HS, JA, and LM contributed to quality checking of the data and the manuscript writing. All authors contributed to the protocol and approved the final manuscript.

\section{Declaration of Conflicting Interests}

The authors declared no potential conflicts of interest with respect to the research, authorship, and/or publication of this article.

\section{Funding}

The authors received no financial support for the research, authorship, and/or publication of this article.

\section{Supplemental Material}

Supplementary material for this article is available online.

\section{References}

Anderson, N. B. (1997). Integrating behavioral and social sciences research at the National Institutes of Health, U.S.A. Social Science \& Medicine, 44, 1069-1071.

Brigham, G. S., Feaster, D. J., Wakim, P. G., \& Dempsey, C. L. (2009). Choosing a control group in effectiveness trials of behavioral drug abuse treatments. Journal of Substance Abuse Treatment, 37, 388-397. doi:10.1016/j.jsat.2009.05.004

Campbell, A. N., Nunes, E. V., Miele, G. M., Matthews, A., Polsky, D., Ghitza, U. E., .. Crowell, A. R. (2012). Design and methodological considerations of an effectiveness trial of a computerassisted intervention: An example from the NIDA Clinical Trials Network. Contemporary Clinical Trials, 33, 386-395. doi:10.1016/j.cct.2011.11.001 
Center for Medical Technology Policy. (2010). Pragmatic Phase 3 Pharmaceutical Trials: Recommendations for the design of clinical trials that are more informative for patients, clinicians, and payers. Retrieved June 9, 2017, from http:/www.cmtpnet.org/ docs/resources/PCT3_EGD.pdf

Chan, A. W., Song, F., Vickers, A., Jefferson, T., Dickersin, K., Gotzsche, P. C., ... van der Worp, H. B. (2014). Increasing value and reducing waste: Addressing inaccessible research. Lancet, 383, 257-266. doi:10.1016/s0140-6736(13)62296-5

Dawson, L., Zarin, D. A., Emanuel, E. J., Friedman, L. M., Chaudhari, B., \& Goodman, S. N. (2009). Considering usual medical care in clinical trial design. PLoS Med, 6, e1000111. doi:10.1371/ journal.pmed.1000111

European Medicines Agency. (2001). ICH Topic E10 Choice of Control Group in Clinical Trials. Retrieved June 9, 2017, from http:// www.ema.europa.eu/docs/en_GB/document_library/Scientific_ guideline/2009/09/WC500002925.pdf

Food and Drug Administration. (2001). Guidance for industry E 10 choice of control group and related issues in clinical trials. Retrieved June 9, 2017, from http:/www.fda.gov/cder/guidance/ index.htm

Hudson, K. L., Lauer, M. S., \& Collins, F. S. (2016). Toward a new era of trust and transparency in clinical trials. Journal of the American Medical Association, 316, 1353-1354. doi:10.1001/jama.2016.14668

Karanicolas, P. J., Farrokhyar, F., \& Bhandari, M. (2010). Practical tips for surgical research: Blinding: Who, what, when, why, how? Canadian Journal of Surgery, 53, 345-348.

Kelly, L. E., Davies, E. H., Saint-Raymond, A., Tomasi, P., \& Offringa, M. (2016). Important issues in the justification of a control treatment in paediatric drug trials. Archives of Disease in Childhood, 101, 962-967. doi:10.1136/archdischild-2016-310644

Kim, J., \& Shin, W. (2014). How to do random allocation (randomization). Clinics in Orthopedic Surgery, 6, 103-109. doi:10.4055/ cios.2014.6.1.103

Mann, H., \& Djulbegovic, B. (2003). Choosing a control intervention for a randomised clinical trial. BMC Medical Research Methodology, 3, 7. doi:10.1186/1471-2288-3-7

Marsden, J., Stillwell, G., Hellier, J., Brown, A. M., Byford, S., Kelleher, M.,... Mitcheson, L. (2017). Effectiveness of adjunctive, personalised psychosocial intervention for nonresponse to opioid agonist treatment: Study protocol for a pragmatic randomised controlled trial. Contemporary Clinical Trials, 53, 36-43. doi:10.1016/j.cct.2016.12.003

McGrath, J. E. ( 1995). Methodology matters: Doing research in the behavioral and social sciences. In R. M. Baecker, J. Grudin, W. A. S. Buxton, S. Greenberg (Eds.), Human-Computer Interaction (pp. 152-169). San Francisco, CA: Morgan Kaufmann Publishers. Pages 152-169 Morgan Kaufmann Publishers Inc. San Francisco, CA, USA (C) 1995

Michie, S., van Stralen, M. M., \& West, R. (2011). The behaviour change wheel: A new method for characterising and designing behaviour change interventions. Implementation Science, 6, 42. doi:10.1186/1748-5908-6-42

Sackett, D. L., \& Wennberg, J. E. (1997). Choosing the best research design for each question. British Medical Journal, 315, 1636.

Samaan, Z., Litke, K., McCabe, K., Dennis, B., Whattam, J., Garrick, L., ... Thabane, L. (2015). A pragmatic pilot randomized trial to investigate the effectiveness of behavioural activation group therapy in reducing depressive symptoms and improving quality of life in patients with depression: The BRAVE pilot trial protocol. Pilot and Feasibility Studies, 1, 39. doi:10.1186/ s40814-015-0034-y

Schulz, K. F., Chalmers, I., Hayes, R. J., \& Altman, D. G. (1995). Empirical evidence of bias. Dimensions of methodological quality associated with estimates of treatment effects in controlled trials. Journal of the American Medical Association, 273, 408-412.

Schwartz, D., \& Lellouch, J. (1967). Explanatory and pragmatic attitudes in therapeutical trials. Journal of Chronic Diseases, 20, 637-648. doi:10.1016/0021-9681(67)90041-0

Smelt, A. F., van der Weele, G. M., Blom, J. W., Gussekloo, J., \& Assendelft, W. J. (2010). How usual is usual care in pragmatic intervention studies in primary care? An overview of recent trials. British Journal of General Practice, 60, e305-e318. doi:10.3399/ bjgp10X514819

World Medical Association. (1997). Declaration of Helsinki: Recommendations guiding physicians in biomedical research involving human subjects. Journal of the American Medical Association, 277, 925. doi:10.1001/jama.1997.03540350075038 